\title{
Workshop report: are diet-induced changes of practical importance in nutrition?
}

\author{
By M. I. GURR, National Institute for Research in Dairying, Shinfield, Reading \\ $R G 2 g A T$
}

The function of a workshop is to provide a forum for detailed discussion on specialized problems and, if possible, to emerge with some clear answers and recommendations about future action. It is an occasion on which scientists can discuss their most preliminary and untested results openly, and even the most reticent can make their contributions to the discussion in the knowledge that the proceedings will not be published in detail or subjected to critical scrutiny by referees. Workshops therefore have a form and purpose quite distinct from symposia and this one played an important role in examining and developing several themes that had emerged in the Society's preceding symposium on 'Nutritional Aspects of Membrane Structure and Function'. The purpose of this brief review is not to provide a detailed account of the workshop discussions but to indicate to those Society members, who could not attend, the most important points to emerge from the workshop.

The plan followed for this workshop was to identify four topics for discussion and to invite, several weeks in advance of the meeting, individuals to prepare a short introductory presentation on each of these topics with the aim of initiating discussion. During the Society's main meeting, immediately before the workshop, others were approached and asked to be prepared to contribute at appropriate stages in the proceedings. All contributors were asked to use no more than two or three slides and to keep their remarks brief, i.e. the workshop proceedings should not drift into a series of formal lectures.

The topics addressed were:

(I) To what extent is diet important in bringing about changes in membrane structure and function that have physiological significance? (Discussion leader: $\mathrm{K}$. Wahle.)

(2) Which membranes are most or least susceptible to dietary influence? (Discussion leader: D. A. York.)

(3) What are the implications for membrane processes and which are the most important (e.g. glucose transport, insulin receptors, other ligand-receptor interactions)? (Discussion leader: $\mathrm{H}$. Tepperman.)

(4) How will dietary guidelines, such as those suggested by the National Advisory Council on Nutrition Education (NACNE), influence these processes? (Discussion leader: M. A. Crawford.)

The first speaker in the symposium, Dr Lee, had introduced us to the current concepts of membrane architecture, the methods by which it is investigated and 
the problems of interpretation of information gained by these various methods in terms of membrane function. The basic concept is that a number of physiological or biochemical processes occurring in membranes may depend for their optimal functioning on the composition and physical characteristics of the membrane. What aspect of membrane architecture is important, how do we measure it and how is it affected by diet? An important point emerging from the workshop discussion was that much (though not all) of the evidence for environmentallyinduced changes in membrane function comes from measurements in vitro and as nutritionists we must ask about their relevance in vivo.

A great deal of attention has been focussed on the property of membrane 'fluidity' and it has often been assumed that this property (however defined) overrides all else. Symposium papers presented by Drs Lee and Houslay demonstrated the need to re-examine this assumption critically and this theme was further developed in the workshop. Several factors contribute to fluidity (e.g. total acyl chain unsaturation, position of double bonds in fatty acid molecules, position of fatty acids on the glycerol backbone of phospholipids, changes in polar headgroups of lipids, cholesterol:phospholipid values, lipid-protein interactions) and any one of these may by itself influence some physiological functions but not others. Few investigators measure all these contributing factors of membrane architecture simultaneously and there should be more general awareness by nutritional biochemists of the complexity of membrane molecular interactions.

Experiments were quoted, in which animals fed on diets rich in polyunsaturated fats had increased membrane acyl chain unsaturation but no change in fluidity because of a compensating effect of an increase in cholesterol: phospholipid. One could not necessarily conclude that because fluidity was unchanged, there would be no influence on some physiological property of the membranes since the function of some enzymes or receptors may be influenced specifically and independently by local changes in sterol or acyl chain composition. Nutritionally-induced changes in the environment of membranes (e.g. availability of fatty acids, hormones) may influence membrane function not by effecting direct compositional changes, but by influencing the activities of membrane enzymes that are themselves capable of affecting membrane architecture (e.g. desaturases, acyl transferases, phospholipases). Different cells and tissues renew themselves at different rates. The turnover of membrane components, lipids and proteins can be very different in, for example, brain and intestine, yet evidence was provided that even tissues with a slow turnover can be influenced by nutritional changes, especially during development. Evidence was also provided for dietary influences, particularly of fat (absolute amount and type) and fibre on membrane glycoproteins and on the insulin responsiveness of cells and the reactivity of platelets that can in part be related to adaptations in the plasma membrane. These nutritionally-induced responses are particularly relevant to conditions such as obesity, diabetes and vascular disease that are prevalent in the UK today. It is likely, therefore, that changes in diet of the type recommended by the recent NACNE discussion paper and the Committee on Medical Aspects of Food Policy report on diet and cardiovascular disease will 
influence the development of these disease states in ways that can be directly related to membrane structure and function.

This Society is still in its infancy in relation to the organization of workshops. In my view they can perform a valuable function and it would be well worthwhile to develop a proper policy with regard to the organization and running of workshops. Lessons to be learned from this one are that it is important to have someone specifically designated to make notes of the main points introduced during the discussion. A tape recording could be helpful but someone would have to be prepared to spend a lot of time in transcription. It is quite impossible for the Chairman to steer the workshop efficiently and to take notes as well. Finally, although this workshop was characterized by some first-rate discussion, with very wide participation, it was impossible to draw out clear conclusions at the end because of time restrictions. It finished late on the last afternoon of the meeting when many participants were anxious to get away. Workshops should be programmed with adequate time and not regarded as a secondary feature of the entire meeting. 